\title{
Nódulo pulmonar solitario: ¿Es la medición del tiempo de duplicación volumétrica realmente útil para diferenciar lesiones benignas de malignas?
}

David Ladrón de Guevara H. ${ }^{*}$, José Tomás Weisz C. $^{2}$, Carolina Schröter G. ${ }^{3}$, Fernando Yerovi G. ${ }^{4}$, Evelyn San Martin M. ${ }^{5}$, Natalia Jara $0 .{ }^{6}$

1. Departamento de Radiología, Clínica Las Condes, Santiago, Chile. Profesor Universidad de Chile.

2. Médico. Becario de Programa de formación de especialista en Radiología. Escuela de Postgrado, Facultad de Medicina. Universidad de Chile. Santiago, Chile.

3. Radiólogo cursando Fellowship de PET/CT e imágenes oncológicas de Clínica Las Condes - Escuela de Postgrado, Facultad de Medicina. Universidad de Chile. Santiago, Chile.

4. Médico. Nuclear en estadía de observación en Unidad de PET/CT, Clínica Las Condes, Santiago, Chile.

5. Médico. Becario de Programa de formación de especialista en Radioterapia Oncológica. Escuela de Postgrado, Facultad de Medicina. Universidad de Chile. Santiago, Chile.

6. Médico. Becario de Programa de formación de especialista en Radioterapia Oncológica. IRAM y Universidad Diego Portales. Santiago, Chile.

Solitary lung nodule: is volumetric doubling time assessment really useful for differentiating a malignant from a benign lesion?

\section{Resumen:}

Objetivo: Establecer el valor diagnóstico del tiempo de duplicación (TD) en la evaluación de nódulos pulmonares sólidos. Método: Se revisó las tomografías computadas (TC) de pacientes derivados por nódulo pulmonar (NP) sólido, que contaran con TC comparativo previo o posterior, incluyendo sólo aquellas lesiones con confirmación histológica. Las metástasis fueron excluidas. Los TD fueron calculados con metodología 2D y 3D. Resultados: La muestra correspondió a 71 NP en 60 pacientes (mediana 68 años, 45-92 años, 59\% hombres), 63 de ellos malignos (neoplasias primarias pulmonares) y 8 benignos. No hubo diferencias en las curvas ROC entre TD calculado mediante método $2 D$ vs $3 D$. Para TD de entre 40 y 400 días, la sensibilidad, especificidad y valor predictivo negativo (VPN) fueron de 63,5\%,62,5\% y 17,9\%; y para TD de entre 20 y 600 días, de 80,9\%, 37,5\% y 20,0\%. Conclusión: La medición del TD de un NP es de limitada utilidad al querer descartar su naturaleza maligna.

Palabras clave: Cáncer pulmonar; Imagen 3D; Nódulo pulmonar solitario; Tiempo de duplicación; Tomografía Computada Multidetector.

\footnotetext{
Abstract:

Aim: To establish the diagnostic accuracy of doubling time (DT) assessment in evaluation of solid lung nodules (LN). Method: Computed Tomography (CT) of patients referred for $P N$ were reviewed, selecting those with an available CT for comparingcomparison. We included LN with histologic confirmationconfirmed by histology only. LN histologically proved as metastases were excluded. DT were calculated both by methodology 2D and 3D methods. Results: Seventy oneSeventy-one LN in 60 patients (median 68 y, 45$92 y, 59 \%$ men) were studied, 63 of them malignant (corresponding a primaries primary lung neoplasms), and 8 were benign. There were not no significant differences in ROC curves among DT calculated by $2 D$ or $3 D$ methodology. Sensitivity, specificity and negative predictive value were $63,5 \%, 62,5 \%$ and $17,9 \%$ for DT between 40-400 days; and 80,9\%,37,5\% and 20,0\% for DT between 20-600 days. Conclusion: DT assessment has limited utility for rule outruling out malignant nature of a solid $L N$.

Keywords: Doubling time; Imaging; Lung cancer; Multidetector Computed Tomography; Solitary pulmonary nodule; Three-Dimensional.

Los autores declaran no tener conflictos de interés.

Ladrón de Guevara D., et al. Nódulo pulmonar solitario: ¿Es la medición del tiempo de duplicación volumétrica realmente útil para diferenciar lesiones benignas de malignas? Rev Chil Radiol 2020; 26(4): 133-140.

*Correo electrónico: David Ladrón de Guevara H. / dlg@clc.cl.

Trabajo enviado el 02 de mayo de 2020. Aceptado para publicación el 15 de agosto de 2020.
} 


\section{Introducción}

El estudio no invasivo de los nódulos pulmonares solitarios ha sido una tarea desafiante para el equipo médico en las últimas décadas. El pilar fundamental en su evaluación imagenológica actual es la tomografía computada (TC), que aporta información como la ubicación, tamaño, forma, bordes y densidad de la lesión, características que ayudan a determinar su naturaleza. Otro factor a considerar es su evolución en el tiempo. La estabilidad por dos años de un NP sólido, o bien su disminución de tamaño apoyarían fuertemente un origen benigno'.

El crecimiento de un nódulo entre controles sucesivos hace sospechar malignidad. Sin embargo, muchas lesiones benignas también pueden crecer ${ }^{2}$. Ya que el crecimiento por sí mismo no garantiza malignidad, se ha tratado de caracterizar la velocidad de crecimiento de dichas lesiones desde mediados del siglo $\mathrm{XX}^{2}$, con el propósito de identificar patrones en la dinámica de crecimiento que puedan estar mejor relacionados a malignidad y de esta manera, reconocer precozmente las lesiones malignas y disminuir la intervención innecesaria de nódulos benignos. Además, el estudio de las tasas de crecimiento ha permitido establecer un tiempo prudente de años de seguimiento y un intervalo temporal adecuado entre controles ${ }^{3}$.

Para estimar la velocidad de crecimiento, se ha propuesto el tiempo de duplicación del volumen tumoral, o tiempo de duplicación (TD) 2,3. Teóricamente, la duplicación del número de células de una lesión, conlleva la duplicación de volumen tumoral ${ }^{3,4,5}$. Esto puede ser representado matemáticamente por medio de un modelo de crecimiento exponencial ${ }^{3,6,7}$.

La TC ha hecho posible estimar de manera precisa el volumen de nódulos pulmonares y así poder estimar el TD de forma reproducible. En la práctica, se puede estimar el volumen tumoral por métodos en dos dimensiones o métodos tridimensionales. Las fórmulas para calcular volumen en dos dimensiones, pueden ser ampliamente utilizadas en clínica, estimando el volumen a partir de un diámetro representativo del nódulo. Los métodos en tres dimensiones requieren tomografías de tórax de alta resolución, con grosor de corte no mayor a $1,5 \mathrm{~mm}$ y la disponibilidad de un software capaz de realizar reconstrucción volumétrica del nódulo para estimar correctamente su volumen en un plano tridimensional5,8.

Al comparar ambas formas de calcular el volumen, los métodos tridimensionales parecen ser más consistentes con el resultado histopatológico, dado el crecimiento asimétrico y no siempre redondeado de algunos nódulos ${ }^{5,7,9}$. Esto es especialmente válido para NP pequeños ${ }^{10,11}$, y cobra relativa importancia tomando en cuenta que actualmente los nódulos pulmonares incidentales más frecuentes de en- contrar en TC, son menores a $10 \mathrm{~mm}^{9}$, donde los métodos en dos dimensiones pierden exactitud, en especial si el crecimiento es lento y/o los intervalos entre controles son muy cortos. En estos casos se ha observado que la variabilidad interobservador aumenta con respecto a los métodos tridimensionales, posiblemente por crecimientos submilimétricos difíciles de medir manualmente ${ }^{9,10,12}$. Actualmente, existen herramientas de software cada vez más fáciles de aplicar, que han automatizado la volumetría tridimensional y la hacen más reproducible ${ }^{13,14,15}$. De todas formas, el tiempo de duplicación de un nódulo se puede calcular a partir de volumetrías tanto bidimensionales -basado en un diámetro, dos diámetros, o área del NP- como tridimensionales, tomando en consideración que se debe utilizar el mismo método volumétrico en el seguimiento para calcular los tiempos de duplicación ${ }^{16}$.

En la literatura se menciona un amplio rango de valores del TD para caracterizar los NP, basado en la premisa de que los NP benignos tienen crecimientos o muy rápidos (TD menores de 20 a 40 días) o muy lento (TD mayores de 400 a 600 días), con valores intermedios para los NP malignos ${ }^{7,9,11,13,17,18,19,20,21}$. Sin embargo, la gran mayoría de las series muestran sobreposición en la velocidad de crecimiento de las lesiones, especialmente en relación a NP malignos que se salen de estos márgenes, lo que disminuiría el valor predictivo negativo del TD a tal punto que sería insuficiente para descartar malignidad de un NP en crecimiento ${ }^{9,13,16,19,20}$.

El objetivo de este estudio es establecer el valor diagnóstico del TD en la evaluación de nódulos pulmonares sólidos cuya naturaleza ha sido confirmada mediante histología.

\section{Pacientes y Método}

Selección de pacientes

Se seleccionó prospectivamente aquellas TC de tórax multicorte solicitadas por estudio de NP sólidos que tuvieran uno o más controles tomográficos comparativos, separados por un lapso de al menos 15 días, que mostraran cambios de tamaño del NP en al menos en uno de ellos. De este grupo, se incluyeron sólo las lesiones con confirmación histológica. Los NP en vidrio esmerilado y las metástasis pulmonares fueron excluidas. Aquellos NP estables respecto a su tamaño no fueron considerados en este estudio. Los criterios de inclusión y exclusión se muestran a continuación.

\section{Criterios de inclusión:}

1. Nódulo pulmonar sólido que muestre cambios de tamaño en TC sucesivos.

2. Confirmación histológica del NP.

3. Histología compatible con lesión de origen primario pulmonar, maligna o benigna. 
Criterios de exclusión:

1. NP sólido estable en controles sucesivos de TC.

2. NP subsólido (en vidrio esmerilado o mixto).

3. NP sin confirmación histológica

4. NP con histología compatible con metástasis.

\section{Aspectos técnicos de la TC}

Todos las TC fueron realizadas en tomógrafo multidetector Siemens 64 canales o General Electric 16 canales, adquiriendo cortes finos de 1,0 $\mathrm{mm} \mathrm{1,25}$ $\mathrm{mm}$, respectivamente. En ambos casos se realizó una adquisición volumétrica en inspiración máxima de todo el campo pulmonar. Las imágenes fueron reconstruidas con método iterativo. Para la medición de las lesiones se efectuaron reconstrucciones multiplanares.

Utilizando los cortes finos con ventana de pulmón, se midió el diámetro mayor multiplanar de cada NP en la TC inicial (D1) y TC de control (D2), registrando la fecha de cada examen, y calculando el lapso de tiempo (días) que separó a cada control tomográfica (T2-T1).

El tiempo de duplicación basado en el diámetro (TDD) de cada NP fue calculado utilizando algoritmo de Schwartz ${ }^{6}$ según la fórmula 1 :

$$
\mathrm{TDD}=\frac{\log 2(T 2-T 1)}{3 \log \left(\frac{D 2}{D 1}\right)}
$$

Mediante software Syngo.via 2015 (C Siemens Healthcare $\mathrm{GmbH}$ ), y utilizando las imágenes con ventana de pulmón, dos operadores entrenados midieron de manera semiautomática el volumen de cada uno de los nódulos en estudio, bajo supervisión de un radiólogo experimentado.

El tiempo de duplicación volumétrico (TDV) de cada NP fue calculado utilizando algoritmo de Schwartz ${ }^{6}$ según la fórmula 2 :

$$
\operatorname{TDV}=\frac{\log 2(T 2-T 1)}{\log \left(\frac{V 2}{V 1}\right)}
$$

Siendo V2 el volumen en el tiempo T2, y V1 el volumen en tiempo T1.

\section{Estudio estadístico}

Se construyeron gráficas Box and Whisker y curvas ROC mediante Software MedCalc versión 18.10.2
(MedCalc Software Ltd., Seúl, República de Corea), y se calculó el rendimiento diagnóstico (sensibilidad, especificidad, valor predictivo positivo (VPP), valor predictivo negativo (VPN) y eficiencia) del TDV para diferenciar NP malignos de benignos, utilizando como "gold standard" la histología. La comparación entre TD se realizó mediante test Mann-Whitney. Para evaluar correlación se utilizó test Spearman rank order.

\section{Aspectos éticos}

El presente estudio contó con la aprobación del comité de ética de la institución. Todos los pacientes incluidos en este estudio han adherido y firmado un Consentimiento Informado para realizar investigación utilizando sus imágenes. Para efectos del análisis de los datos y la publicación del estudio, se usó la información codificada. Se protegió la privacidad y confidencialidad de los pacientes del estudio.

\section{Resultados}

La muestra del estudio estuvo constituida por 71 NP en 60 pacientes (mediana 68 años, rango 45-92 años, $59 \%$ hombres), con un tiempo de observación medio de 202 días (18-1892 días). El tamaño, volumen y TDV de los nódulos según su diagnóstico histológico, se muestra en la tabla 1.

La medición de los TD basados en el volumen derivado del diámetro (TDD) y en el volumen calculado por volumetría (TDV) mostraron una significativa correlación (rho: 0,745, p<0,0001), como se muestra en la figura 1. No hubo diferencias significativas entre las curvas ROC de TDD y TDV, con áreas bajo la curva de 0,775 y 0,748 , respectivamente (Figura 2). El mejor valor de corte de la curva ROC fue de un TD de 31 días, con una Sensibilidad, Especificidad y VPN de $95,2 \%, 50,0 \%$ y $57,1 \%$. Para este valor de corte quedaron sin diagnosticar 3 NP malignos (Cáncer pulmonar células pequeñas, Adenocarcinoma y Escamoso).

El grupo histológico cáncer pulmonar no células pequeñas (CPNCP) presentó TDV (mediana 184 días, rango-1.823-3.146 días) significativamente mayor que el de células pequeñas (CPCP) (mediana 35 días, rango 25-119 días) y que los NP benignos (mediana 53 días, rango -112-431 días). Por otro lado, el CPNCP tuvo TDV significativamente menor que los tumores carcinoides (mediana 655 días, rango 410-1.515 días). Las gráficas de Box and Whisker (lineal y logarítmica) de los TDV según el diagnóstico histológico se muestran en la figura 3.

EI TDV de los NP benignos no mostró diferencias significativas con el TDV de los CPCP.

La Sensibilidad, Especificidad y VPN del TDV entre $40-400$ días fue de $63,5 \%, 62,5 \%$ y $17,9 \%$, respectivamente. Para el rango $20-400$ días fue de $69,8 \%, 50,0 \%$ y $17,4 \%$; y para el rango $20-600$ días fue de $80,9 \%, 37,5 \%$ y $20,0 \%$ (Tabla 2 ). 
Tabla 1. Tamaño (diámetro mayor), Volumen y TD volumétrico de 71 NP sólidos según diagnóstico histológico.

\begin{tabular}{|c|c|c|c|c|c|c|}
\hline & $\mathrm{n}$ & $\begin{array}{c}\text { Tamaño } \\
(\mathrm{mm})\end{array}$ & $\begin{array}{l}\text { Volumen } \\
\text { (cc) }\end{array}$ & $\begin{array}{l}\text { TD Vol } \\
\text { (días) }\end{array}$ & $\mathrm{p}$ & $\mathrm{p}$ \\
\hline CPNCP & 54 & $7,6(2,5-41,1)$ & $0,251(0,007-27,9)$ & $184(-1822-3146)$ & * & $\infty \&$ \\
\hline AD & 37 & $6,8(2,5-41,1)$ & $0,230(0,022-27,9)$ & $193(-1822-3146)$ & & \\
\hline ES & 5 & $19,9(9,1-21,7)$ & $0,828(0,397-3,83)$ & $146(-390-348)$ & NS & \\
\hline CG & 3 & $6,3(2,5-22,7)$ & $0,143(0,007-4,03)$ & $119(38-159)$ & NS & \\
\hline NOS & 9 & $7,0(2,7-25,4)$ & $0,260(0,014-7,52)$ & $104(46-1921)$ & NS & \\
\hline СPCP & 5 & $7,7(7,6-9,9)$ & $0,241(0,120-0,45)$ & $35(25-119)$ & ${ }^{\star} 0,0041$ & $\dagger$ \\
\hline Carcinoide & 4 & $7,6(7,0-13,3)$ & $0,266(0,163-1,09)$ & 655 (410-1515) & 0,0141 & $\infty 0,0141$ \\
\hline Benignos & 8 & $13,0(3,6-25,8)$ & $0,956(0,031-6,40)$ & $53(-112-431)$ & 0,0081 & †NS \& 0,0221 \\
\hline
\end{tabular}

CPNCP: Cáncer pulmonar no células pequeñas; AD: Adenocarcinoma; ES: Carcinoma Escamoso; CG: Carcinoma de células grandes; NOS: CPNCP de histología no especificada; CPCP: carcinoma pulmonar células pequeñas.
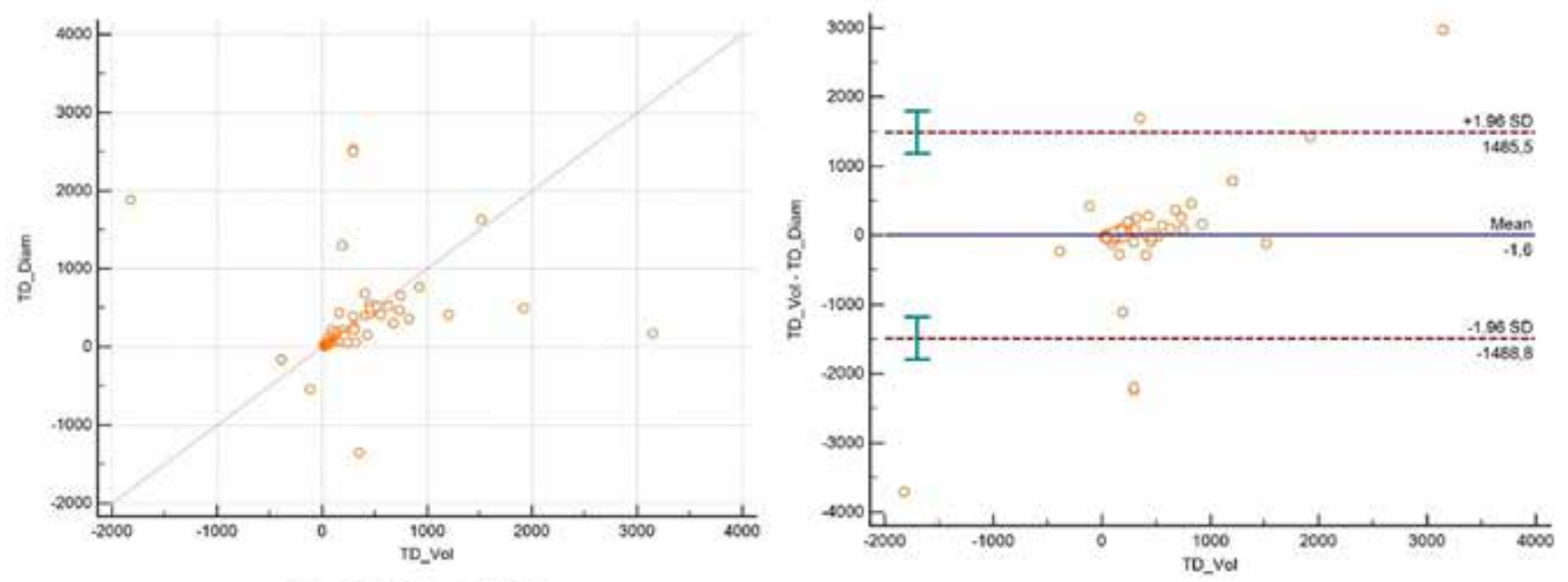

Rho: 0,745 p $<0,0001$

Figura 1: Correlación entre los tiempos de duplicación calculados en base al volumen derivado del diámetro (TDD) y en base al volumen calculado directamente por volumetría (TDV). A izquierda un gráfico de correlación lineal de ambas medidas y a derecha un gráfico de Bland-Altman.

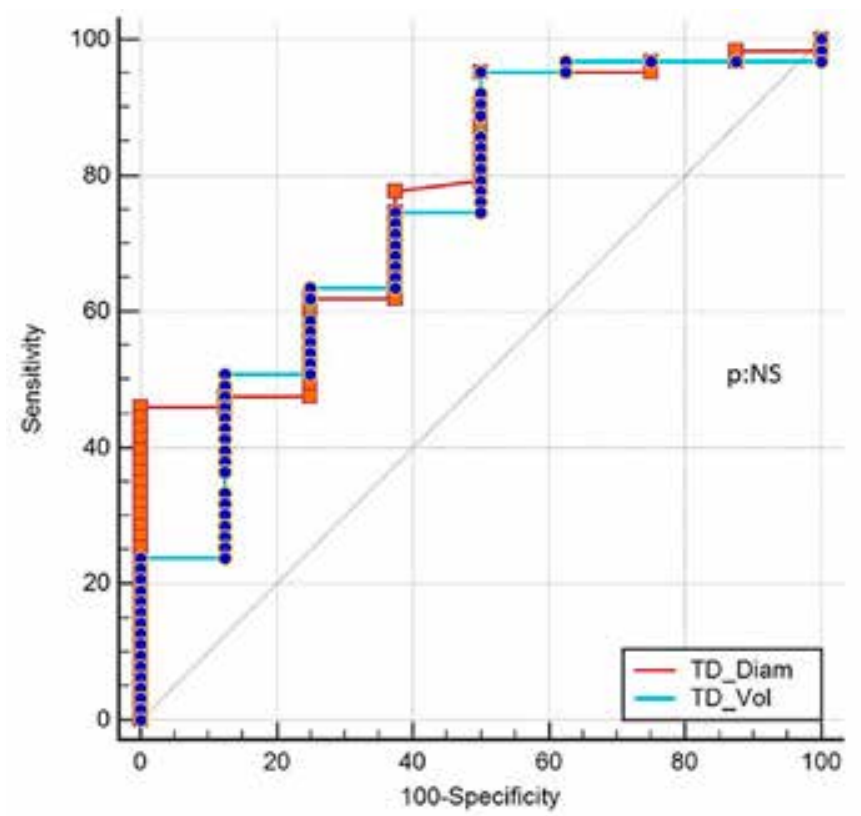

Figura 2: Curva ROC comparativa entre TDD y TDV. 


\section{TDV según histología}

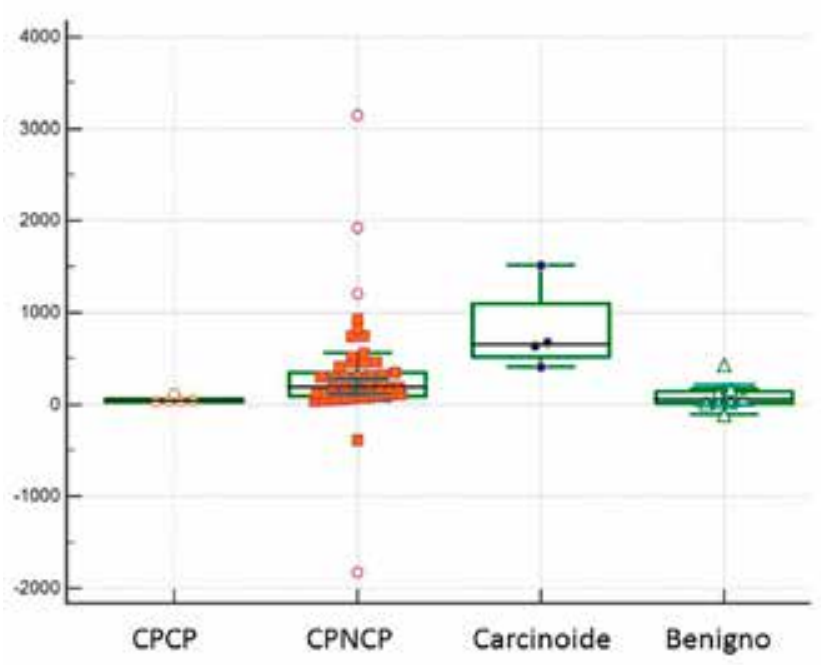

Box and Whisker lineal

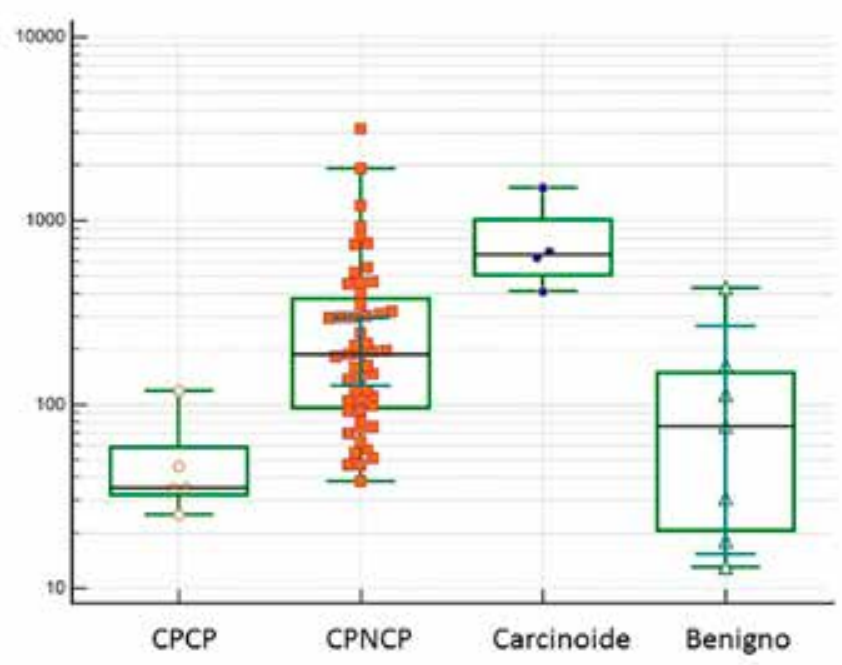

Box and Whisker logaritmica

Figura 3: Gráficas de Box and Whisker lineal (izquierda) y logarítmica (derecha) del TDV según diagnóstico histológico.

Tabla 2. Rendimiento diagnóstico del TDV para tres valores de corte basados en la literatura.

\begin{tabular}{|c|c|c|c|}
\hline & TDV $40-400$ & TDV 20 - 400 & TDV $20-600$ \\
\hline VP & 40 & 44 & 51 \\
\hline FP & 3 & 4 & 5 \\
\hline VN & 5 & 4 & 3 \\
\hline FN & 23 & 19 & 12 \\
\hline Sensibilidad & $63,5 \%$ & $69,8 \%$ & $80,9 \%$ \\
\hline Especificidad & $62,5 \%$ & $50,0 \%$ & $37,5 \%$ \\
\hline VPP & $93,0 \%$ & $91,7 \%$ & $91,1 \%$ \\
\hline VPN & $17,9 \%$ & $17,4 \%$ & $20,0 \%$ \\
\hline Exactitud & $63,4 \%$ & $67,6 \%$ & $76,1 \%$ \\
\hline $\mathrm{LR}+$ & 1,69 & 1,39 & 1,29 \\
\hline LR - & 0,58 & 0,60 & 0,51 \\
\hline
\end{tabular}

VP: Verdaderos positivos; FP: Falsos positivos; VN: Verdaderos negativos; FN: Falsos Negativos; VPP: Valor predictivo positivo; VPN: Valor predictivo negativo; LR: Likelihood ratio.

Si consideramos el valor de corte clásico del TD $<40$ días para estimar un NP como benigno, quedan sin diagnosticar 6 cánceres pulmonares de crecimiento rápido ( $3 \mathrm{CPCP}, 1$ Adenocarcinoma, 1 Escamoso, y 1 NOS), es decir el $10,2 \%$ de los cánceres pulmonares de la muestra (6/59) (Figura 4).

Si consideramos el valor de corte clásico para
NP benignos de TD > 400 días, se escapan al diagnóstico 13 cánceres pulmonares con crecimiento lento (12 adenocarcinomas, 1 NOS), es decir el 22\% de los cánceres pulmonares (13/59).

Ya que los 4 tumores carcinoides presentaron TDV $>400$ días, el rango teórico de benignidad de NP con TD $<40$ días ó $>400$ días sólo permite diagnosticar el 63,5\% de los NP malignos (40/63). 


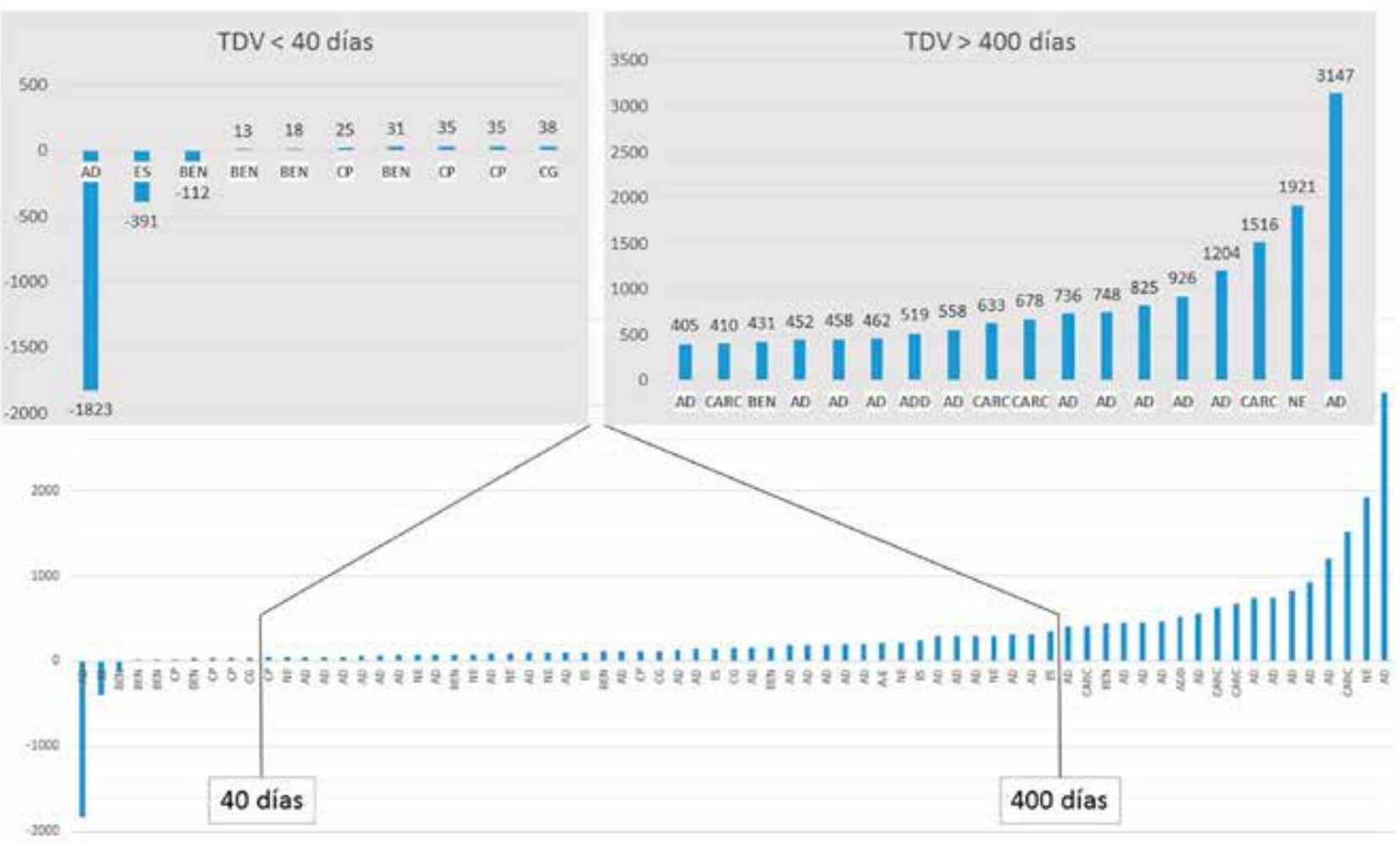

Figura 4: Tiempo de duplicación volumétrico en días del grupo total de nódulos pulmonares según diagnóstico histológico.

\section{Discusión}

La velocidad de crecimiento de los NP depende de múltiples factores. El primero a considerar es la naturaleza de la lesión, ya que las lesiones primarias pulmonares tienen un comportamiento diferente a las neoplasias secundarias pulmonares, generalmente con crecimientos más acelerados de estas últimas 6 . Por otro lado, el crecimiento de las metástasis pulmonares es de difícil observación, ya que una vez diagnosticadas, su evolución natural se verá modificada por cualquier tratamiento sistémico administrado. En otras palabras, la TC comparativa de control casi siempre será tomada bajo efectos de quimioterapia, inmunoterapia, terapias dirigidas u otras. Debido a esto, y a la diferente biología de las lesiones secundarias, este estudio se basó sólo en la observación de lesiones primarias pulmonares.

Otro factor a considerar, es el aspecto del NP a la TC. Aquellos NP con densidad en vidrio esmerilado, que corresponden generalmente a lesiones malignas primarias pulmonares de menor grado histológico y agresividad, generalmente adenocarcinomas no invasivos o mínimamente invasivos, suelen presentar crecimiento lepídico y muy baja tasa de replicación celular $^{3}$, con crecimiento no necesariamente exponencial y TD que pueden exceder tranquilamente los 1.000 días ${ }^{7,18,22}$. Además, el tamaño no es el único factor a considerar en estas lesiones, ya que el cambio de densidad también es clave en su seguimiento'. Esto pudiera ser aplicado en parte a nódulos subsólidos mixtos, aunque su naturaleza invasiva les imprime un carácter de mayor agresividad que suele implicar un aumento en su velocidad de crecimiento ${ }^{6,13}$. Sin embargo, la literatura es escasa respecto al TD de estas lesiones mixtas.

Los NP malignos sólidos suelen ser lesiones más agresivas que aquellas en vidrio esmerilado puro $^{3,13}$, aunque su tasa de replicación celular es extremadamente variable. La heterogeneidad de líneas celulares tumorales en combinación con la respuesta inmune propia de cada paciente, resulta en una gran dispersión de los TD en estas lesiones ${ }^{6}$. Los NP sólidos malignos también pueden presentar necrosis, licuefacción o cavitación, lo que alteraría el modelo de crecimiento exponencial y consecuentemente la estimación del tiempo de duplicación ${ }^{7}$. Lindell et al reportaron disminución de tamaño en 4 de 48 tumores pulmonares, todos ellos adenocarcinoma ${ }^{20}$. Igual experiencia publico Honda et al, con VTD negativos en 5 de 51 cánceres pulmonares, todos ellos adenocarcinomas ${ }^{19}$. Esto fue visto en tres NP malignos de nuestra muestra, que disminuyeron su tamaño en las TC de seguimiento, produciendo TD negativos.

Una alta precisión en el cálculo del volumen de la lesión es un importante pre-requisito en la objetivación de crecimiento y cálculo del TD de los NP ${ }^{15}$. Para esto se requiere una serie de condiciones: 1) Contar con 
exámenes de tomografía computada multidetector de corte fino $(\leq 1,5 \mathrm{~mm})$ con adecuada adquisición y reconstrucción de imágenes. La radiografía de tórax no permite una medición adecuada de las lesiones. 2) Un método confiable y reproducible de estimar el volumen.

El tiempo de duplicación obtenido varía considerablemente respecto al método de medición del volumen del NP, basado en el diámetro (2D), en el área (2D) o directamente en volumetría (3D) $)^{5,15}$. EI diámetro mayor del NP ha sido la medida estándar en la monitorización de los cambios de tamaño ${ }^{9}$. Otros autores proponen la medición del diámetro mayor y el diámetro perpendicular en el mismo eje ${ }^{3}$. Sin embargo, la medición de diámetros o áreas es por definición bidimensional, lo que presupone que la lesión crece uniformemente en todas las direcciones ${ }^{5}$, algo que en la práctica no ocurre. Por otro lado, la medición manual implica una variabilidad intrínseca, por cuanto la reproducibilidad del método manual de medición no es perfecta ${ }^{9}$. Revel et al obtuvieron índices de repetitividad de entre 1,3 y 1,7 al medir nódulos pulmonares pequeños, concluyendo que la medición bidimensional de las lesiones no es confiable al evaluar NP menores de $20 \mathrm{~mm}^{10}$. Koike et al demostraron una mayor reproducibilidad del cálculo volumétrico 3D asistido por computador en comparación al cálculo manual del diámetro en NP malignos $^{13}$. Debido a esto, la medición volumétrica $3 \mathrm{D}$ sería, en teoría, más exacta que aquella generada en base a mediciones 2D. Esto está demostrado en estudios que utilizan mediciones automatizadas en fantomas de lesiones esférica y lobuladas, que presentan idéntico diámetro, pero diferentes volúmenes ${ }^{8}$.

Si revisamos la escasa literatura acerca de la utilidad diagnóstica del TD, podemos encontrar que una parte importante de ella se basa en estudios planares, realizando mediciones de NP basado en radiografías, publicaciones antiguas llevadas a cabo en la era pre $\mathrm{TC}^{6,21,22,23}$. Otras series que contaron con la técnica tomográfica, calcularon los TD basados en un diámetro representativo, varios de ellos mezclando NP sólidos con subsólidos ${ }^{3,17,20}$, que sabemos tienen una biología y crecimiento diferente. En resumen, la bibliografía acerca del TD en NP sólidos utilizando volumetría es escasa, y siempre con un número acotado de pacientes $5,7,9,13,16$. Consideramos que la alta selección de la población en estudio, y la aplicación de volumetría como sustrato para medir el TD, es una fortaleza de nuestro estudio.

Asumiendo que el TDV calculado cumple todos los requerimientos mencionados, aún surge la interrogante de cuál es su utilidad en el estudio de los NP. Los resultados al emplear los valores de corte clásicos expuestos en la tabla 2 , determinan un número inaceptablemente alto de falsos negativos, aún aquél de TDV entre 20 y 600 días que determina 12
NP malignos que son erróneamente diagnosticados como benignos, en una muestra de $63 \mathrm{NP}$ malignos totales.

En nuestra casuística se obtuvo una correlación aceptable entre ambos métodos de medición del volumen, y no encontramos diferencias significativas en el rendimiento diagnóstico del TD medido utilizando el diámetro mayor (2D) vs la volumetría (3D), ambos con áreas bajo la curva similares en la curva ROC. Sin embargo, no medimos la reproducibilidad de ambos métodos, lo que constituye una limitación de nuestro estudio.

Existen datos que consideran el TD como factor independiente de mal pronóstico ${ }^{22}$ y además se ha correlacionado el TD de los nódulos pulmonares, con estudios funcionales con PET FDG, observando que tumores de crecimiento rápido, presentan una mayor concentración del radiotrazador ${ }^{4,24}$. Sin embargo, hacen falta más estudios para poder determinar si valores de SUVmax determinados logran predecir el tiempo de duplicación de un nódulo.

\section{Conclusión}

El estudio de la utilidad diagnóstica del TD requiere una serie de condiciones para ser considerado válido en la práctica clínica actual, teniendo especial cuidado en la adecuada selección del tipo de NP a estudiar, y en la metodología para medir el volumen y el TD del NP. Aún en condiciones ideales de medición, el TD volumétrico es de limitada utilidad al querer descartar lesiones malignas (bajo VPN), y por lo tanto no es recomendable como método aislado para evitar estudios invasivos en NP sólidos.

\section{Referencias}

1. MacMahon H, Naidich DP, Goo JM, Lee KS, Leung ANC, Mayo JR, et al. Guidelines for Management of Incidental Pulmonary Nodules Detected on CT Images: From the Fleischner Society 2017. Radiology. 2017; 284: 228-243.

2. Nathan $\mathrm{MH}$, Collins VP, Adams RA. Differentiation of Benign and Malignant Pulmonary Nodules by Growth Rate. Radiology 1962; 79: 221-232.

3. Hasegawa M, Sone S, Takashima S, Li F, Yang ZG, Maruyama $Y$, et al. Growth rate of small lung cancers detected on mass CT screening. Br J Radiol. 2000; 73: 1252-1259.

4. Duhaylongsod FG, Lowe VJ, Patz EF, Vaughn AL, Coleman RE, Wolfe WG. Lung tumor growth correlates with glucose metabolism measured by fluoride-18 fluorodeoxyglucose positron emission tomography. Ann Thoracic Surg. 1995; 60: 1348-1352.

5. Yankelevitz DF, Reeves AP, Kostis WJ, Zhao B, Henschke Cl. Small Pulmonary Nodules: Volumetrically Determined Growth Rates Based on CT Evaluation. Radiology. 2000; 217: 251-256.

6. Schwartz M. A Biomathematical approach to clinical tumor growth. Cancer 1961; 14: 1272-1294.

7. Li J, Xia T, Yang X, Dong X, Liang J, Zhong N, et al. 
Malignant solitary pulmonary nodules: assessment of mass growth rate and doubling time at follow-up CT. J Thorac Dis. 2018; 10 (Suppl 7): S797-S806.

8. Ravenel JG, Leue WM, Nietert PJ, Miller JV, Taylor KK, Silvestri GA. Pulmonary Nodule Volume: Effects of Reconstruction Parameters on Automated Measurements-A Phantom Study. Radiology. 2008; 247: 400-408.

9. Revel MP, Merlin A, Peyrard S, Triki R, Couchon S, Chatellier G, et al. Software Volumetric Evaluation of Doubling Times for Differentiating Benign Versus Malignant Pulmonary Nodules. AJR. 2006; 187: 135-142.

10. Revel M-P, Bissery A, Bienvenu M, Aycard L, Lefort C, Frija G. Are Two-dimensional CT Measurements of Small Noncalcified Pulmonary Nodules Reliable? Radiology. 2004; 231: 453-458.

11. Borghesi A, Michelini S, Scrimieri A, Golemi S, Maroldi R. Solid Indeterminate Pulmonary Nodules of Less Than $300 \mathrm{~mm}^{3}$ : Application of Different Volume Doubling Time Cut-offs in Clinical Practice. Diagnostics. 2019; 9: 62. doi:10.3390/diagnostics9020062.

12. Revel M-P, Lefort C, Bissery A, Bienvenu M, Aycard L, Chatellier G, et al. Pulmonary Nodules: Preliminary Experience with Three-dimensional Evaluation. Radiology. 2004; 231: 459-466.

13. Koike W, Iwano S, Matsuo K, Kitano M, Kawakami K, Naganawa S. Doubling time calculations for lung cancer by three-dimensional computer-aided volumetry: Effects of inter-observer differences and nodule characteristics. J Med Imaging Radiat Oncol. 2014; 58: 82-88.

14. Marten K, Engelke C. Computer-aided detection and automated CT volumetry of pulmonary nodules. Eur Radiol. 2007; 17: 888-901.

15. Wormanns D, Kohl G, Klotz E, Marheine A, Beyer F, Heindel W, et al. Volumetric measurements of pulmonary nodules at multi-row detector CT: in vivo reproducibility. Eur Radiol. 2004; 14: 86-92.

16. Quint LE, Cheng J, Schipper M, Chang AC, Kalemkerian
G. Lung lesion doubling times: values and variability based on method of volume determination. Clinical Radiology. 2008; 63: 41-48.

17. Wang JC, Sone S, Feng L, Yang ZG, Takashima S, Maruyama $Y$, et al. Rapidly growing small peripheral lung cancers detected by screening CT: correlation between radiological appearance and pathological features. Br J Radiol. 2000; 73: 930-937.

18. Eisenbud Quint LE, Cheng J, Schipper M, Chang AC, Kalemkerian $\mathrm{G}$. Lung lesion doubling times: values and variability based on method of volume determination. Clin Radiol. 2008; 63: 41-48.

19. Honda O, Johkoh T, Sekiguchi J, Tomiyama N, Mihara $\mathrm{N}$, Sumikawa $\mathrm{H}$, et al. Doubling time of lung cancer determined using three-dimensional volumetric software: Comparison of squamous cell carcinoma and adenocarcinoma. Lung Cancer. 2009; 66: 211-217.

20. Lindell RM, Hartman TE, Swensen SJ, Jett JR, Midthun DE, Tazelaar HD, et al. Five-year lung cancer screening experience: CT appearance, growth rate, location, and histologic features of 61 lung cancers. Radiology. 2007; 242: 555-562.

21. Geddes DM. The natural history of lung cancer: a review based on rates of tumour growth. Br J Dis Chest. 1979; 73: 1-17.

22. Usuda K, Saito Y, Sagawa M, Sato M, Kanma K, Takahashi S, et al. Tumor doubling time and prognostic assessment of patients with primary lung cancer. Cancer. 1994; 74: 2239-2244.

23. Fujimura S, Suda S, Yamauchi A. Tumor doubling time and PPD skin test reactivity in resectable lung cancer. J Jpn Lung Cancer Soc. 1979; 19: 135-142.

24. Ruilong Z, Daohai X, Li G, Xiaohong W, Chunjie W, Lei T. Diagnostic value of 18F-FDG-PET/CT for the evaluation of solitary pulmonary nodules: a systematic review and meta-analysis. Nuclear Medicine Communications. 2017 enero; 38(1): 67-75. 